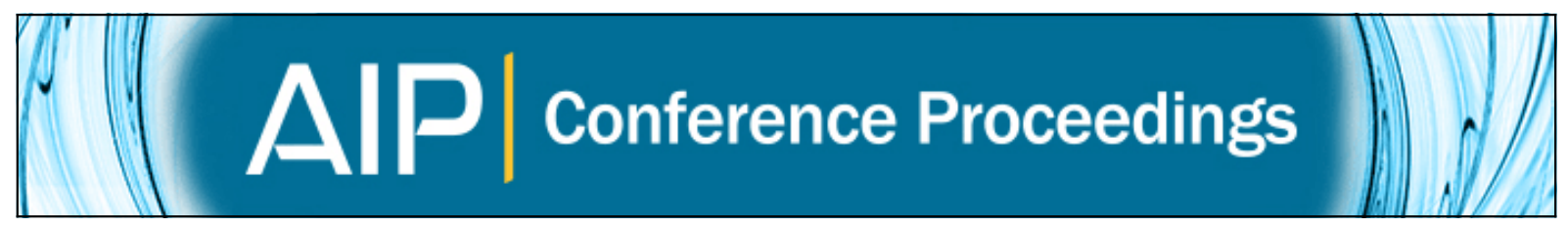

\title{
Development of a melting model for meteors
}

Bruno Dias, Federico Bariselli, Alessandro Turchi, Aldo Frezzotti, Philippe Chatelain, and Thierry Magin

Citation: AIP Conference Proceedings 1786, 160004 (2016); doi: 10.1063/1.4967661

View online: http://dx.doi.org/10.1063/1.4967661

View Table of Contents: http://scitation.aip.org/content/aip/proceeding/aipcp/1786?ver=pdfcov

Published by the AIP Publishing

\section{Articles you may be interested in}

Modeling the melting of supported clusters

Appl. Phys. Lett. 88, 133110 (2006); 10.1063/1.2187950

New Developments in Plasma Arc Melting

J. Vac. Sci. Technol. 9, 1344 (1972); 10.1116/1.1317036

Meteors

Phys. Today 18, 118 (1965); 10.1063/1.3047402

Meteors: Proceedings of a Symposium on Meteor Physics

Phys. Today 9, 31 (1956); 10.1063/1.3060002

New model predicts altitude at which meteors will explode Phys. Today 


\title{
Development of a Melting Model for Meteors
}

\author{
Bruno Dias ${ }^{1, \text { a) }}$, Federico Bariselli ${ }^{1}$, Alessandro Turchi ${ }^{1}$, Aldo Frezzotti $^{2}$, Philippe \\ Chatelain $^{3}$ and Thierry Magin ${ }^{1}$
}

\author{
${ }^{1}$ von Karman Institute for Fluid Dynamics, Chaussée de Waterloo, 72, B-1640 Rhode-St-Genèse, Belgium \\ ${ }^{2}$ Politecnico di Milano, Dipartimento di Scienze e Tecnologie Aerospaziali, Via La Masa 34, 20156 Milan, Italy \\ ${ }^{3}$ Institute of Mechanics, Materials and Civil Engineering (iMMC), Université catholique de Louvain (UCL), B-1348 \\ Louvain-la-Neuve, Belgium \\ a)barros@vki.ac.be
}

\begin{abstract}
Meteor phenomenon is a frequent event happening on planet Earth. Due to the high entry velocities of these objects, the surface of the material undergoes extreme heat loads. Since the material is mainly composed by several oxides, eventually, the surface temperature will overcome the melting point. In this study we propose a melting model, in order to understand the material behavior, coupled with a flow solver. A detailed study of the flow around the stagnation streamline is also presented.
\end{abstract}

\section{Introduction}

From 50 to 100 tonnes of meteoroids enter Earth's atmosphere every day. Entry velocities can vary between $11.5 \mathrm{~km} / \mathrm{s}$ and $72 \mathrm{~km} / \mathrm{s}$, and these strong hypersonic conditions lead to extreme temperatures on the surface of these objects. Since most of the meteoroids are composed by oxides, such as $\mathrm{SiO}_{2}$ and $\mathrm{MgO}$, the extreme heat loads at the surface promotes the material to melt and eventually to evaporate. Overall, this process leads to a huge amount of mass deposition in the upper atmosphere and is the main reason of the growing interest in studying the ablation of these space objects. During the meteoroid disintegration, ablation products interact with the ionized gas surrounding the meteoroids and are driven to the wake giving a signature that can be detected by radars. The detection of meteors aims to answer questions such as: "What is the real meteor incoming flux?" and "What is the influence the mass deposition into the upper atmosphere for aeronomy purposes?"

Recent efforts have been made by the Belgian Institute for Space Aeronomy in order to predict velocity and trajectory of the meteors, by means of an innovative technique based on radio waves. The Belgian Radio Meteor Stations (BRAMS) experiment, consists in a series of receivers spread all over Belgium to collect and standardize the meteor observation data. In brief, a signal sent by a transmitter, is first reflected by the free electrons in the trail of the meteor, and then captured by the ground receivers (Forward Scattering technique) allowing for the study of the meteor atmospheric entry. One of the main objective of this project is the computation of the meteoroid flux densities. However, this quantity is hardly quantifiable from the lone radar observation, and it is necessary to rely on comparison with numerical models to have an estimate of it. In this scenario, the necessity of having the capability to numerically reproduce the meteor entry finds its room.

Current ablation models [1], are based on the Knudsen-Langmuir. These kind of models disregard any melting of the material considering only evaporation once the melting temperatures is reached.

The development of melting models was initially conducted in the 50's by Bethe and Adams [2]. The use of glassy materials as protection of spacecraft vehicles led to deep study of the melting phenomenon. More recently, with the advances of the metallurgic industry [3], there was a necessity to improve such models. Moreover it has been observed from the meteorites recovered in the ground [4], an importance in considering this phenomenon.

The study of meteor ablation mentioned above, are used to understand the mass deposition in the atmosphere. However, since they were conceived to study the ablation of small particles in the upper atmosphere, they usually rely on a zero dimensional approach and lack of an accurate treatment of the particle interaction with the atmosphere from the fluid dynamic point of view. Therefore, for meteoroids big enough to survive this first phase of the atmospheric 
entry, a different approach has to be sought. Moreover the study of material melting and its possible removal by mechanical forces is often neglected.

In this work we propose to study the meteor ablation with a numerical approach similar to those used in the aerospace community to model the gas-surface interaction over TPS materials. A material solver has been developed in order to solve the Stefan problem and understand better the melting of meteors. Furthermore, the material solver has been coupled with a 1-D Stagnation-Line CFD solver with an integrated evaporation boundary condition and part of a meteor-entry trajectory has been computed in a coupled fashion.

\section{Flow field radiation}

At the range of velocities typical of meteor phenomena, radiation becomes highly significant, the intense light observed during a meteor entry is mainly due to the radiation of the air and metallic species, where the latter are coming from ablation products.

By solving the radiative transfer equations (RTE) one can determine the spectral radiative intensity, therefore, the radiative source terms, $\mathcal{P}^{r a d}$ and $\mathcal{P}^{r a d}, V E$ and $\dot{\omega}_{i}^{\text {rad }}$. The production or destruction of species, $\dot{\omega}_{i}^{\text {rad }}$, due to radiative processes such as photoionization and photodissociation and the radiative source terms are shown in detail by Soucasse et al. [5]. Solving the RTE by conventional methods such as Line-by-Line (LBL) becomes computationally very expensive for complex molecular spectra.

Recently, Lamet et al. [6] presented a hybrid statistical narrow band model (HSNB) showing an accurate description of the radiative flux with low CPU costs for non-ablative materials. Later Scoggins et al. [7], extended this approach for ablative TPS material.

The feature of the HSNB is that if the radiative mechanisms are statistically uncorrelated one can divide the spectra into narrow bands and compute the intensity in terms of averages. The different mechanism can be separated in two 3 contributions: optically thick molecular systems, optically thin molecular systems and continua, atomic lines. Due to the weak spectral density of atomic lines, this contribution has to be solved by LBL methods. Scoggins et al. [8] presents an atomic spectral mesh to optimize to the LBL method for atomic lines. Details about the HSNB can be found in Refs. [6, 7].

\section{Gas-Surface Interaction}

The evaporation of a melted layer can be modeled by limiting the control volume to an infinitesimal thin lamina where the surface is located. Typically mass conservation for the generic species $i$ can be written in terms of a surface mass balance (SMB)[9]. To solve this balance a suitable model to compute the surface reactions is needed, which in general can be solved by heterogeneous finite-rate chemistry. However, this approach is not always feasible due to the lack of specific data when more exotic surfaces are considered. In this case, a possible solution is then to consider the heterogeneous mixture (hot gas phase and material surface) to be in thermochemical equilibrium at the local conditions of pressure and temperature. As shown by Bianchi, Nasuti, and Martelli [10], when such an equilibrium approach is considered, it is useful to write the SMB in terms of elements which reads as:

$$
J_{k, w}+\dot{m}_{e v a p} y_{k, s}=(\rho u)_{w} y_{k, w} \quad \forall k \in \varepsilon
$$

where $(\rho u)_{w}$ is the gas blowing, $y_{k, w}$ is the mass fraction of element $k$ in the gaseous mixture at the wall, $y_{k, s}$ is the elemental mass fraction of the solid, $\dot{m}$ is the mass blowing rate and $J_{k, w}$ is the elemental mass diffusion. By summing Eq. 1 over all elements, one obtains:

$$
\dot{m}_{\text {evap }}=(\rho u)_{w}
$$

and finally the mass blowing rate can be computed as:

$$
\dot{m}_{\text {evap }}=\frac{J_{k, w}}{\left(y_{k, w}-y_{k, s}\right)} \quad \forall k \in \varepsilon
$$

where the elemental composition of the solid phase, $y_{k, s}$, is known and the elemental composition of the gas can be computed by solving a multiphase equilibrium problem. Once the equilibrium gas composition is known and the mass 
blowing rate, $\dot{m}$, is computed, the surface blowing velocity can be easily obtained as:

$$
u_{w}=\frac{\dot{m}_{\text {evap }}}{\rho_{w}}
$$

where $\rho_{w}$ is the density of the mixture in gas phase at the wall. Finally the recession velocity, $\dot{s}$, can be computed as:

$$
\dot{s}=\frac{\dot{m}_{\text {evap }}}{\rho_{s}}
$$

being $\rho_{s}$ the density of the solid phase.

The removal of liquid layer due to mechanical forces was first derived by Bethe and Adams [2]. By assuming the steady-state incompressible governing equations, the tangential velocity was derived by the authors as:

$$
v=\tau_{\text {flow } / \text { melt }} \int_{0}^{\delta} \frac{d r}{\mu(T)}+\frac{1}{R} \frac{\partial P}{\partial \theta} \text { flow/melt } \int_{0}^{\delta} \frac{r}{\mu(T)} d r
$$

where by knowing the molten layer thickness, $\delta$, Eq. 5 can be easily integrated. The liquid layer viscosity, for glassy materials, is a function of temperature expressed by:

$$
\mu(T)=\exp ([(a / T)-b])
$$

where the coefficients $a$ and $b$ can be found in Bronshten [4] for ordinary chondrites. Finally the corresponding mass flux due to mechanical removal is obtained by:

$$
\dot{m}_{\text {melt }}=\rho_{\text {melt }} v
$$

The total mass removal of the liquid layer has then contributions from both the evaporation and the mechanical forces.

Analogously one can consider a balance of energy fluxes entering and exiting the surface. The surface energy balance (SEB) is described by:

$$
q_{c o n v}+q_{\text {rad,in }}+\left(\dot{m}_{\text {evap }}+\dot{m}_{\text {melt }}\right) h_{c}=\epsilon \sigma T_{w}^{4}+\dot{m}_{\text {evap }} h_{w}+k \frac{\partial T}{\partial r}+\dot{m}_{\text {melt }} h_{c}
$$

where the terms on the left side represent the incoming convective heat flux (conductive heat flux and diffusive heat flux), shock layer radiation and the energy of the solid due to mass loss. On the right side the fluxes leaving the surface are represented by the re-radiation of the material, the energy of the gas composition at the wall, the material heat conduction and energy lost due to liquid removal. By solving this non-linear equation, one can estimate the surface temperature $T_{w}$.

\section{Numerical tools}

The flow governing equations are solved through a CFD code where the SMB boundary condition, explained the in previous section, is implemented. The flow properties are computed with the VKI physico-chemical library $\left(\right.$ Mutation $\left.^{++}\right)$. In order to study the effect of melting and estimate the liquid layer thickness, a 1D material code has been built where SEB and liquid mass removal are solved.

\section{Stagnation-Line Code CFD Solver}

The 1D Stagnation-Line solver developed at the VKI by Munafò [11], resolves the Navier-Stokes equations along the stagnation line of spherical or cylindrical bodies, where an illustration is shown by Fig. 1. This model leads to an efficient way to calculate hypersonic flows with low computational costs. In this code the three-dimensional NavierStokes equations are reduced to a one-dimensional approximation for the stagnation streamline by a system called "Dimensionally Reduced Navier Stokes Equations" (DRNSE). The spatial discretization is done by finite volume method and the time integration is computed by a Backward-Euler method. The stagnation-line equations read as: 


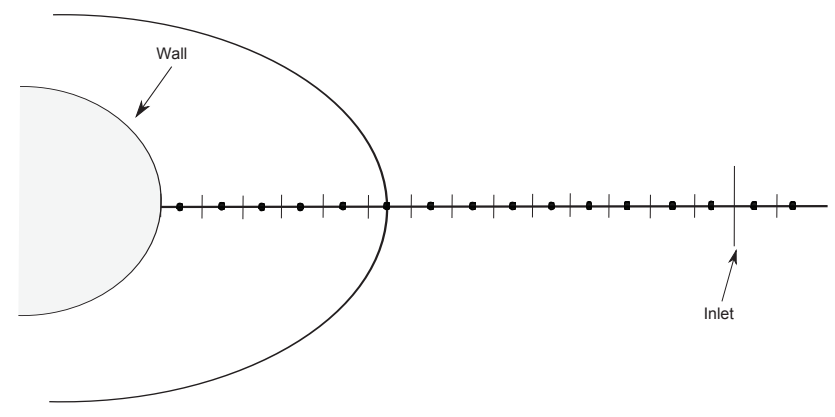

FIGURE 1: 1D Stagnation-Line illustration

$$
\frac{\partial}{\partial t} \mathbf{U}+\frac{\partial}{\partial r} \mathbf{F}^{\mathbf{i n v}}+\frac{\partial}{\partial r} \mathbf{F}^{\mathbf{v i s}}+\frac{\mathbf{G}^{\text {inv }}+\mathbf{G}^{\mathbf{v i s}}}{r}=\mathbf{S}^{\mathbf{c}}+\mathbf{S}^{\mathbf{r}}
$$

Where $\mathbf{U}$ denotes the volume-specific balance quantities, $\mathbf{F}^{\text {inv }}$ are the inviscid fluxes, $\mathbf{F}^{\mathbf{v i s}}$ the viscous terms, $\mathbf{S}^{\mathbf{c}}$ and $\mathbf{S}^{\mathbf{r}}$ are respectively the chemical and radiative source terms:

$$
\begin{aligned}
\mathbf{U} & =\left[\rho_{i}, \rho u, \rho v, \rho E, \rho e^{V E}\right]^{T} \\
\mathbf{F}^{\text {inv }} & =\left[\rho_{i} u, \rho u^{2}+p, \rho u v, \rho u H, \rho u e^{V E}\right]^{T} \\
\mathbf{F}^{\mathbf{v i s}} & =\left[\mathrm{J}_{r i}, \bar{\tau}_{r r}, \bar{\tau}_{r \theta}, q_{r}+\bar{\tau}_{r r} u, q_{r}^{V E}\right]^{T} \\
\mathbf{G}^{\text {inv }} & =\left[2 \rho_{i}(u+v), 2 \rho u(u+v), 3 \rho v(u+v)-2 \bar{p}, 2 \rho H(u+v), \rho e^{V E}\right]^{T} \\
\mathbf{G}^{\mathbf{v i s}} & =\left[\mathrm{J}_{r i}, 2\left(\bar{\tau}_{r r}-\bar{\tau}_{\theta \theta}+\bar{\tau}_{r \theta}\right),-\bar{\tau}_{\theta \theta}+3 \bar{\tau}_{r \theta}, 2\left(q_{r}+\bar{\tau}_{r r} u+\bar{\tau}_{\theta \theta} u+\bar{\tau}_{r \theta} v\right), 2 q_{r}^{V E}\right]^{T} \\
\mathbf{S}^{\mathbf{c}} & =\left[\dot{\omega}_{i}^{\mathbf{c}}, 0,0,0, \Omega^{V E}\right]^{T} \\
\mathbf{S}^{\mathbf{r}} & =\left[\dot{\omega}_{i}^{\mathbf{r a d}}, 0,0, \mathcal{P}^{r a d}, \mathcal{P}^{r a d, V E}\right]^{T}
\end{aligned}
$$

The variables $\mathbf{G}^{\text {inv }}$ and $\mathbf{G}^{\mathbf{v i s}}$ are inviscid and viscous source terms arising from the DRNSE transformation, respectively. The therm $\rho e^{V E}$ stands for vibrational-electronic-electron energy. Details regarding the radial heat fluxes, $q_{r}$ and $q_{r}^{V E}$ as well the energy transfer source term, $\Omega^{V E}$ are given by Scoggins et al. [7].

\section{MUlticomponent Thermodynamic And Transport properties for IONized plasmas in C++ (Mutation $^{++}$)}

The closure of the multi-temperature Navier-Stokes equations is done by computing thermodynamic and transport properties. Mutation ${ }^{++}$is a library built at the VKI by Scoggins [12] where thermodynamic properties are computed by the Rigid Rotor Harmonic Oscillator model while transport properties are derived by the Chapman-Enskog expansion from Kinetic Theory [13].

\section{Multiphase Equilibrium}

Accurate chemical equilibrium solutions are necessary to have a proper estimation of the therm $y_{k, w}$ in Eq. 2. As shown in Table 1, the meteor under investigation (Ordinary Chondrites) is composed by three elements.

Recently an equilibrium multiphase solver based on the Gibbs function continuation method was developed at VKI by Scoggins and Magin [14] and implemented inside the library Mutation ${ }^{++}$. The solver proved to be very robust by converting the minimization of the mixture Gibbs free energy into an initial value problem which can be easily integrated. Furthermore, the solver has the capability of imposing any constraint in order to treat multiple surface components, which becomes a useful approach, when the actual evolution of the surface is unknown. 
TABLE 1: Meteors surface properties

\begin{tabular}{|c|c|c|c|c|}
\hline \multicolumn{2}{|c|}{ Composition $^{\mathrm{a}}$} & \multicolumn{2}{|c|}{ Elemental composition $^{a}$} & Density $\left[\mathrm{kg} / \mathrm{m}^{3}\right]$ \\
\hline $\mathrm{SiO}_{2}$ & 0.606 & $\mathrm{Si}$ & 0.232 & \\
\hline $\mathrm{MgO}$ & 0.394 & $\begin{array}{l}\mathrm{Mg} \\
\mathrm{O}\end{array}$ & $\begin{array}{l}0.152 \\
0.616\end{array}$ & 3000 \\
\hline
\end{tabular}

${ }^{a}$ Mole fractions are shown.

In brief the ratio between each element in the condensed phase is always respected in the gaseous products. By doing this, it can be ensured that in the case of ablation all the surface elements are changing phase with the same rate and the surface composition is always clearly defined.

\section{Melting material solver}

The study of meteor melting is essentially a Stefan problem. By solving the heat equation in spherical coordinates,

$$
\rho c_{p}(T) \frac{\partial T}{\partial t}=\frac{1}{r^{2}} \frac{\partial}{\partial r}\left(r^{2} k \frac{\partial T}{\partial r}\right)
$$

one can compute the temperature throughout the material and the melted layer can be estimated. Since the material presents different phases, a thermodynamic property such as the $c_{p}$ has different values for the corresponding phases. Therefore in order to define accurately the molten interface one must build $c_{p}$ as a function of temperature. This means that the $c_{p}$ of the solid region $\left(1050 \mathrm{~J} \mathrm{~kg}^{-1} \mathrm{~K}^{-1}\right)$ is different from the liquid $\left(1650 \mathrm{~J} \mathrm{~kg}^{-1} \mathrm{~K}^{-1}\right)$ and the corresponding solid/liquid interface will be represented as a discontinuity in the property. Moreover for simplicity it will be assumed symmetry between the polar and azimuthal angles transforming the domain into one dimensional. This hypotheses was posed by Bronshten [4], where is stated that, due the rotation of the meteor, the surface temperature tends to be uniform. To solve Eq. 8 an unsteady finite differences solver was built. The time integration is done by an explicit method. Since the material is recessing due to evaporation and mechanical removal, the change of the domain must be considered. This is done by building an updated domain at each time step and remapping the solution on the new mesh.

\section{Solvers coupling strategy}

As mentioned before, a coupling between material and flow solver is needed. Once the steady state solution of the flow is reached, convective heat flux and evaporation rate are exchanged with the material solver. Then the material solver is responsible for solving the transient solution in a predefined macro time step where the SEB is computed at each internal time. Once the desired time step is reached the surface temperature is provided to the flow solver for the next trajectory point.

For the presented analysis an implicit coupling was chosen [15]. This type of coupling allows for time varying properties into the material solver. In other words, after obtaining the wall temperature from the material solver, the stagnation-line code is run a first time to compute the initial guess of mass blowing rate and convective heat flux. These quantities are linearly interpolated with those computed at the end of the previous trajectory point. The time varying quantities are assigned again to the material solver providing a new solution for the wall temperature. This procedure is repeated until converge is reached, i.e, once the wall temperature difference between two iterations is less than $50 \mathrm{~K}$.

\section{Test case and results}

We considered a $1 \mathrm{~cm}$ radius ordinary chondrite with a constant velocity of $15 \mathrm{~km} \mathrm{~s}^{-1}$ along the trajectory. The trajectory was defined to start at $60 \mathrm{~km}$, ending at $50 \mathrm{~km}$ altitude, thus, the total trajectory time for this conditions corresponds to $0.67 \mathrm{~s}$. This trajectory was divided into 10 points where the solvers coupling methodology mentioned before was employed. 


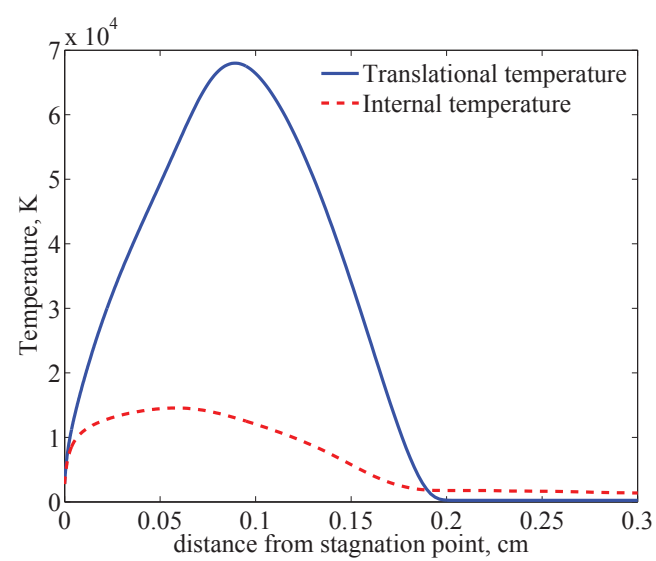

(a) $60 \mathrm{~km}$

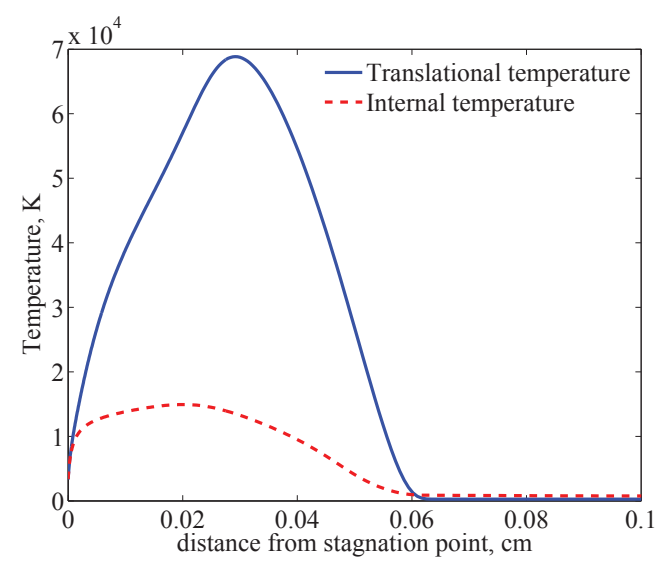

(b) $50 \mathrm{~km}$

FIGURE 2: Temperature profile along the stagnation streamline

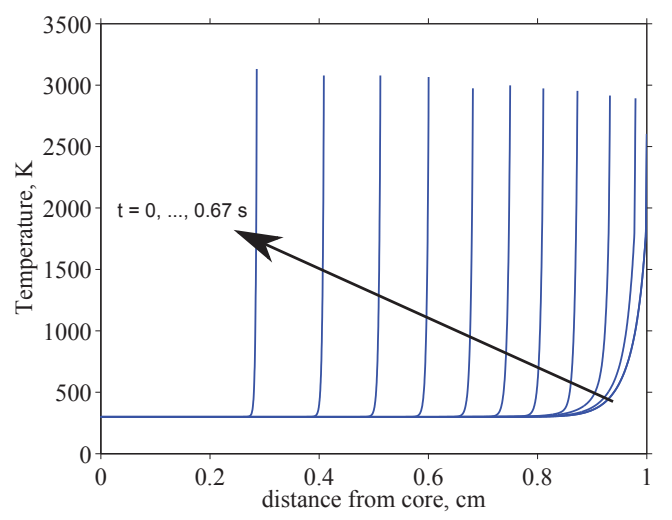

FIGURE 3: In-depth material temperature along trajectory

The ablation species injected into the flow field were considered frozen due to the lack of specific data on their chemical reaction rates. Differently, air species were modeled with finite rate chemistry with reaction rates taken from Park, Jaffe, and Partridge [16]. A two-temperature model was used for these simulations, i.e., $\mathrm{T}=\mathrm{T}_{\text {rot }}$ and $\mathrm{T}_{\text {vib }}=\mathrm{T}_{\text {ele }}$.

Figure 2 shows the temperature profile along the stagnation streamline at 60 and $50 \mathrm{~km}$ altitude. From Fig. 2(a) is visible the shock roughly located at $0.2 \mathrm{~cm}$ from the surface, being very diffused due to the altitude conditions. The maximum translation temperature reaches values around $70000 \mathrm{~K}$ and drops abruptly to the surface. The intense non-equilibrium between translational and internal temperature is also visible around the shock layer. Comparing the previous figure with Fig. 2(b), the latter presents a reduced shock layer thickness. This is due to the increase of the free-stream pressure avoiding the shock layer at $60 \mathrm{~km}$ to be as diffused as at the previous altitude. From both figures it is also visible a thermal non-equilibrium in the free-stream region. This process occurs due to photoionization reactions in that region. In other words, due to strong radiative field, the photons cross the shock wave colliding with the neutral species in the free-stream.

Figure 3 shows the temperature profile inside the material for the different trajectory points. It is visible that the surface temperature, around $3000 \mathrm{~K}$, remains almost constant along the trajectory. Since the recession of the material is much higher than the thermal diffusion speed of the material, it is noticeable a steep temperature profile around the surface, leading the core of the meteor to remain unchanged at $300 \mathrm{~K}$. One can notice, from the temperature profiles, that the recession of the material due to evaporation and mechanical removal, is around $0.75 \mathrm{~cm}$ for the full trajectory.

Then molten thickness along the trajectory is shown in Fig. 4. It is visible a very thin layer mostly due to the high 


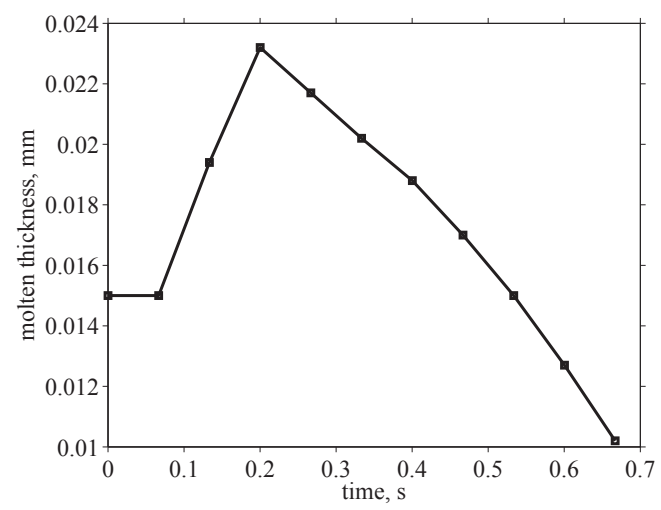

FIGURE 4: Interface location along the trajectory

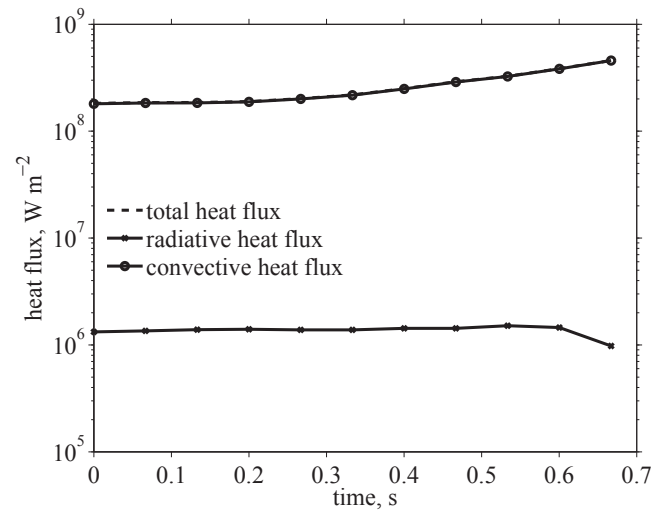

(a) Flow heat flux at the surface

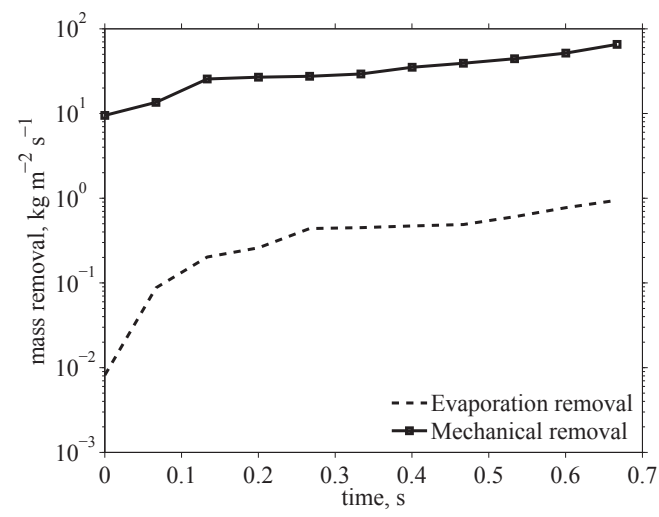

(b) Material mass removal

FIGURE 5: Heat flux and mass removal

mass removal by mechanical forces as shown by Fig. 5(b). The biggest contribution of the mechanical removal is due to the stress forces, i.e. the term $\tau_{\text {flow/melt }}$ in Eq. 5 . The mass removal due to evaporation remains limited due to fact that the surface temperature does not reach the material sublimation point.

Finally Fig. 5(a) shows the contributions of heat flux to the surface. The high temperature gradients next to surface drives the convective heat flux to be the major contribution to the total heat flux. The radiative heat flux is rather small when compared to the convective contribution mostly due to the small shock layer thickness.

\section{Conclusion}

A model to study the meteor ablation at low altitude has been proposed and implemented. The chosen parameters (trajectory, entry velocity and material size) were selected arbitrarily with the purpose of presenting a different approach from the one proposed by the state of the art. The model takes the clue from the strategy used to evaluate the gas-surface interaction on the heat shields of re-entry capsules. In order to study the material response under the harsh entry environment, a material solver was built with the main objective of studying the removal of the molten layer of the meteor. Considering the fact that the material solver requires information from the flow, namely the boundary layer, a coupling with a flow solver was required.

A trajectory $\left(60 \mathrm{~km}\right.$ to $50 \mathrm{~km}$ altitude) of a $1 \mathrm{~cm}$ ordinary chondrite meteor entering at $15 \mathrm{~km} \mathrm{~s}^{-1}$ was studied. Under this conditions it has been observed that the main responsible for the of mass removal is the mechanical removal leading to a very thin molten layer along the trajectory.

It is important to mention that at this conditions double ionized species should be considered, such as $\mathrm{N}^{++}$and 
$\mathrm{O}^{++}$, which may allow a decrease of the translational temperature in the shock layer. Also the radiation of the ablation products was not considered, the absorption of these species may act as cooling effect of the shock layer.

Further comparison of the proposed melting model with experimental results are needed. The model developed for melting can be used to study larger meteors at altitudes where the continuum assumption is valid, such as fireballs. Most of the BRAMS observations are conducted above $80 \mathrm{~km}$ altitude where rarefied gas effects prevail. The melting model can be coupled to a DSMC method to study these conditions [17].

\section{ACKNOWLEDGMENTS}

This research is sponsored by the Belgian Research Action Through Interdisciplinary Networks "METRO: Meteor trajectories and origins". Research of B. Dias is sponsored by fellowship from the "Fonds pour la Formation la Recherche dans l'Industrie et dans l'Agriculture.".

\section{REFERENCES}

[1] T. Vondrak, J. M. Plane, S. Broadley, and D. Janches, Atmospheric Chemistry and Physics 8, 7015-7031 (2008).

[2] H. Bethe and M. C. Adams, Journal of the Aerospace Sciences 26, 321-328 (1959).

[3] A. Faghri and Y. Zhang, Transport Phenomena in Multiphase Systems (Elsevier, Burlington, MA, 2006).

[4] V. A. Bronshten, Physics of Meteoric Phenomena (D. Reidel Pub. Co., 1983).

[5] L. Soucasse, J. Scoggins, P. Rivire, T. Magin, and A. Soufiani, Journal of Quantitative Spectroscopy and Radiative Transfer 180, 55-69 (2016).

[6] J. Lamet, P. Rivière, M. Perrin, and A. Soufiani, Journal of Quantitative Spectroscopy and Radiative Transfer 111, 87-104 (2010).

[7] J. Scoggins, L. Soucasse, P. Riviere, S. Soufiani, and T. Magin, in 45th AIAA Thermophysics Conference (Dallas, Texas, 2015) AIAA 2015-3112.

[8] J. Scoggins, L. Soucasse, P. Riviere, S. Soufiani, and T. Magin, "An adaptive hybrid statistical narrow band model for coupled," in Proceedings of 8th European Symposium on Aerothermodynamics for Space Vehicles, May 2015 (ESTEC, Noordwijk, The Netherlands, 2015).

[9] A. Turchi, "A Gas-Surface Interaction Model for the Numerical Study of Rocket nozzle flows over pyrolyzing ablative materials," Ph.D. thesis, Università degli Studi di Roma "La Sapienza" 2013.

[10] D. Bianchi, F. Nasuti, and E. Martelli, Journal of Spacecraft and Rockets 47, 554-562 (2010).

[11] A. Munafò, "Multi-Scale Models and Computational Methods for Aerothermodynamics," Ph.D. thesis, Ecole Central Paris 2014.

[12] J. Scoggins, "Development of Mutation++: MUlticomponent Thermodynamics And Transport properties for IONized gases library in C++," VKI PhD Symposium (2013).

[13] T. E. Magin and G. Degrez, Journal of Computational Physics 198, 424-449 (2004).

[14] J. Scoggins and T. Magin, Combustion and Flame 162, 4514-4522 (2015).

[15] P. Schrooyen, "Numerical simulation of aerothermal flows through ablative thermal protection systems," Ph.D. thesis, Université catholique de Louvain 2016.

[16] C. Park, R. Jaffe, and H. Partridge, Journal of Thermophysics and Heat Transfer 15, 76-90 (2001).

[17] F. Bariselli, E. Torres, and T. Magin, in 30th International Symposium on Rarefied Gas Dynamics (Victoria, Canada, 2016).

[18] M. Campbell-Brown, D. Koschny, J. Zender, and O. Witasse, Astronomy \& Astrophysics 418, 751-758 (2004).

[19] Z. Ceplecha, J. Borovicka, W. Elford, D. ReVelle, R. Hawkes, V. Porubcan, and M. Simek, Space Science Reviews 84, 327-471 (1998).

[20] C. Park, in 43rd AIAA Thermophysics Conference (New Orleans, Louisiana, 2012) AIAA 2012-3000.

[21] J. Zinn, D. Judd, and D. ReVelle, Advances in Space Research 33, 1466-1474 (2004). 